\title{
Temperature-dependent structural heterogeneity in calcium silicate liquids
}

\author{
C. J. Benmore, ${ }^{1, *}$ J. K. R. Weber, ${ }^{1,2}$ M. C. Wilding, ${ }^{3}$ J. Du, ${ }^{4}$ and J. B. Parise ${ }^{5}$ \\ ${ }^{1}$ X-ray Science Division, Advanced Photon Source, Argonne National Laboratory, Argonne, Illinois 60439, USA \\ ${ }^{2}$ Materials Development, Inc., Arlington Heights, Illinois 60004, USA \\ ${ }^{3}$ Institute of Mathematical and Physical Sciences, Abersytwyth University, Aberystwyth, Cerdigion SY23 3BZ, United Kingdom \\ ${ }^{4}$ Department of Material Science and Engineering, University of North Texas, Denton, Texas 76203, USA \\ ${ }^{5}$ Mineral Physics Institute and Department of Geosciences, Stony Brook University, Stony Brook, New York 11794-2100, USA
}

(Received 30 September 2010; published 7 December 2010)

\begin{abstract}
$\mathrm{X}$-ray diffraction measurements performed on aerodynamically levitated $\mathrm{CaSiO}_{3}$ droplets have been interpreted using a structurally heterogeneous liquid-state model. When cooled, the high-temperature liquid shows evidence of the polymerization of edge shared Ca octahedra. Diffraction isosbestic points are used to characterize the polymerization process in the pair-distribution function. This behavior is linear in the hightemperature melt but exhibits rapid growth just above the glass transition temperature around $1.2 T_{\mathrm{g}}$. The heterogeneous liquid interpretation is supported by molecular-dynamics simulations which show the $\mathrm{CaSiO}_{3}$ glass has more edge-shared polyhedra and fewer corner shared polyhedra than the liquid model.
\end{abstract}

DOI: 10.1103/PhysRevB.82.224202

PACS number(s): 61.20.-p, 61.05.cp, 64.70.P-

\section{INTRODUCTION}

Bulk liquids are often regarded as homogeneous materials partly due to their inherently disordered nature and also because many of their properties can be modeled by a uniform continuum. When liquids are supercooled, a dynamic heterogeneity occurs around the crossover from "normal" liquid to "complex" solidlike properties ${ }^{1}$ as the glass transition is approached. This coincides with a similar crossover from mode coupling theory at a temperature of $T=1.2 T_{\mathrm{g}}$ between a primarily "ergodic" liquid to a mainly "nonergodic" glassy regime. ${ }^{2}$ Unlike the dynamic heterogeneity however, structural heterogeneity in a liquid at the atomic level is difficult to establish and to our knowledge there has been no definitive evidence for structural heterogeneity occurring in the pair-distribution functions of glass-forming liquids. ${ }^{2,3}$ This is despite the analysis of quenched glass structures that indicate atomic to nanometer scale structural heterogeneity must occur in the liquid state. ${ }^{4-7}$

Silica is classified as a strong liquid with an Arrhenius viscosity-temperature relation and only subtle differences occur between the liquid and glass structures. ${ }^{8,9}$ In contrast $\mathrm{CaO}-\mathrm{SiO}_{2}$ liquids can be considered fragile based on viscosity measurements ${ }^{10,11}$ and consequently larger structural rearrangements are likely to be necessary to form a glass. In this study we combine containerless aerodynamic levitation, high-energy $\mathrm{x}$-ray diffraction and molecular-dynamics (MD) simulations to interpret and follow the structural evolution of calcium silicate liquids from high temperatures to the glassy state. Using the concept of diffraction isosbestic points we suggest that at temperatures $>1000{ }^{\circ} \mathrm{C}$ liquid $\mathrm{CaSiO}_{3}[$ melting point $1540{ }^{\circ} \mathrm{C}$ (Ref. 10)] can be considered a heterogeneous two component liquid. Using this model we observe a linear growth of $\mathrm{CaO}_{6}$ octahedra in the high-temperature liquid as it is cooled. However as the glass transition temperature is approached the polymerization of edge-shared $\mathrm{CaO}_{6}$ octahedra increases rapidly.

\section{EXPERIMENTAL}

Six compositions between 40 and 75 mol \% $\mathrm{CaO}$ were investigated in the liquid state. The materials were made from calcium carbonate and silica powders that were mixed, calcined and consolidated into spheres in a laser hearth. Compositions containing less than $60 \mathrm{~mol} \% \mathrm{CaO}$ formed glasses when cooled. In this work we present only the results only for $\mathrm{CaSiO}_{3}$ in detail as the other glass-forming compositions all showed a similar behavior. The high-energy $\mathrm{x}$-ray diffraction experiments on the calcium silicate liquids were performed using an aerodynamic levitator installed on beamline $11 \mathrm{ID}-\mathrm{C}$ at the Advanced Photon Source (APS), USA. ${ }^{12}$ The temperature of the melts was controlled using a $240 \mathrm{~W}$ $\mathrm{CO}_{2}$ laser beam. The experiment was performed in transmission geometry with a $1 \mathrm{~mm} \times 1 \mathrm{~mm}$ x-ray beam of wavelength $0.1088 \AA$ and diffraction patterns on isothermal melts were collected using a Mar345 image plate detector mounted orthogonal to the X-ray beam. Samples of 2-3 mm in diameter were made from binary $(\mathrm{CaO})_{\mathrm{x}}\left(\mathrm{SiO}_{2}\right)_{(1-\mathrm{x})}$ compositions containing $x=40,50,60,70$, and $80 \mathrm{~mol} \% \mathrm{CaO}$. The sample lost $\sim 1 \%$ of its initial mass during the hightemperature levitation experiments in pure oxygen. Microprobe analysis on the recovered samples indicated that the composition change was $<1 \%$.

Calcium silicate liquids were studied at static temperatures ranging from $\sim 800$ to $\sim 1900{ }^{\circ} \mathrm{C}$ in approximately $100{ }^{\circ} \mathrm{C}$ increments. The data were reduced using the software FIT2D (Ref. 13) and the x-ray structure factors, $S(Q)$, obtained up to $Q \sim 19 \AA^{-1}$ using the program PDFGETX2 (Ref. 14) see Fig. 1(a). The apparent temperature of the levitated sample was measured using an optical pyrometer that operated at a wavelength of $0.650 \mu \mathrm{m}$ as described previously. ${ }^{12}$ The estimated errors associated with the temperature measurement arising from the emissivity correction and temperature gradients that occur in the high temperature melts are $\pm 25{ }^{\circ} \mathrm{C}$ for $T>1000{ }^{\circ} \mathrm{C}$ and $\pm 50{ }^{\circ} \mathrm{C}$ at lower temperatures approaching the glass transition temperature.

Fast, transient cooling measurements were made on selected samples using a Perkin Elmer model $1621 \mathrm{x}$-ray area detector. This arrangement enabled a measurement of structure to be made in a period of $200 \mathrm{~ms}$ and a sequence of measurements were made as the liquid drop cooled from about 2000 to $600{ }^{\circ} \mathrm{C}$. An optical pyrometer (Chino Corpo- 

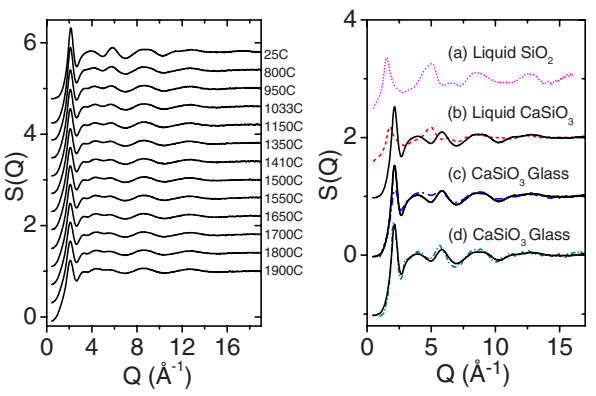

FIG. 1. (Color online) Left: measured x-ray structure factors for $\mathrm{CaSiO}_{3}$ over a temperature range that spans the superheated and supercooled liquid and the glass. Right: comparison of our $\mathrm{CaSiO}_{3}$ data with previously measured $\mathrm{x}$-ray structure factors for glassy and liquid $\mathrm{CaSiO}_{3}$ and $\mathrm{SiO}_{2}$ from the literature. (a) Shows liquid $\mathrm{SiO}_{2}$ (scaled by 0.5) from (Ref. 9) (magenta dotted line). (b) Our measured liquid structure factor for $\mathrm{CaSiO}_{3}$ at $1650{ }^{\circ} \mathrm{C}$ (black solid line) is compared to that measured by Waseda and Toguri (Ref. 15) at $1600{ }^{\circ} \mathrm{C}$ (red dashed line). The solid black lines in (c) and (d) are the measured glass structure factors of $\mathrm{CaSiO}_{3}$ at $25{ }^{\circ} \mathrm{C}$ (this study) compared to Taniguchi et al. (Ref. 16) [(c) blue long dashed-dotted line] and Mead and Mountjoy (Ref. 17) [(d) green short dasheddotted line]. The curves are offset for clarity.

ration, Tokyo, Japan) operating at $10 \mathrm{~Hz}$ was sighted onto the levitated drop in the region where it was heated by the laser beam and the x-ray beam intersected the droplet. The effective wavelength of the pyrometer was $0.9 \mu \mathrm{m}$ with a spot size of $0.6 \mathrm{~mm}$. The apparent temperatures measured with the pyrometer were corrected using an emissivity value of 0.92 , estimated by assuming that the drop was opaque at the measurement wavelength. This assumption is met at temperatures above $\sim 1000{ }^{\circ} \mathrm{C}$ but as the drop cools below this temperature it gradually becomes transparent and its effective emissivity decreases. As a result of this effect, the apparent temperature appears to decrease faster than it would at constant emissivity. For example at $800{ }^{\circ} \mathrm{C}$, if the emissivity were 0.5 rather than 0.92 the sample temperature would appear to be $45^{\circ} \mathrm{C}$ lower than it would at constant emissivity.

\section{EXPERIMENTAL RESULTS}

Our measured liquid and glass $\mathrm{x}$-ray structure factors are shown in Fig. 1(a) and compared with curves digitized from the literature in Fig. 1(b). The difference between liquid $\mathrm{CaSiO}_{3}$ at $1650{ }^{\circ} \mathrm{C}$ (this study) and $1600{ }^{\circ} \mathrm{C}$ from Ref. 15 is most likely due to a difference in composition, as noted previously. ${ }^{18}$ The preferential evaporation of $\mathrm{CaO}$ over a long (tens of minutes) period of time in the experiment described in Ref. 15 could leave a more silica-rich liquid than that of the $\mathrm{CaSiO}_{3}$ starting material, as suggested by the similarity to pure liquid $\mathrm{SiO}_{2} S(Q)$ as illustrated in Fig. 1(b). The shape of our glass $\mathrm{x}$-ray structure factors are similar to those measured by Taniguchi et al. ${ }^{16}$ except in the low- $Q$ region [Fig. 1(c)] but are in good agreement with the more recent data of Mead and Mountjoy ${ }^{17}$ [Fig. 1(d)].

The real space pair-distribution functions $D(\mathrm{r})$ $=4 \pi \rho r[G(\mathrm{r})-1]$ with the bulk density removed ${ }^{20}$ were obtained for liquid $\mathrm{CaSiO}_{3}$ at $1900{ }^{\circ} \mathrm{C}$ and $1033{ }^{\circ} \mathrm{C}$ and are

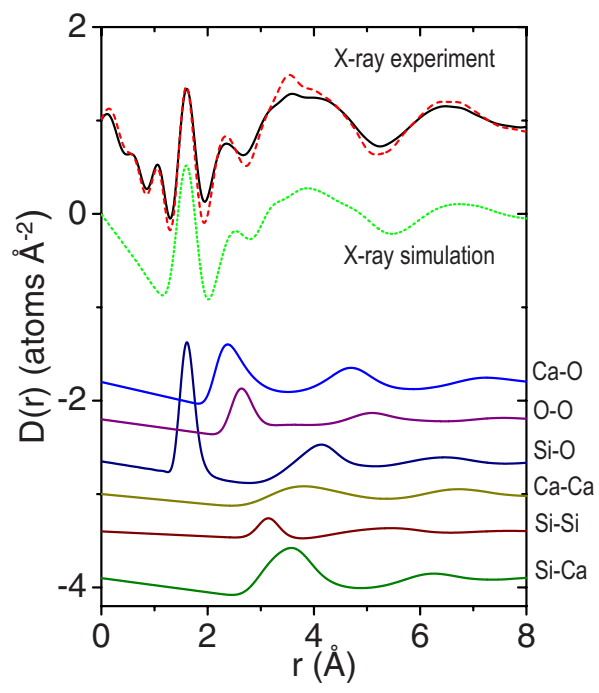

FIG. 2. (Color online) Top: differential distribution functions, $D(r)$, for liquid $\mathrm{CaSiO}_{3}$ at $1900{ }^{\circ} \mathrm{C}$ (solid black line) and $1033{ }^{\circ} \mathrm{C}$ (dashed red line). Middle: molecular-dynamics simulation of $\mathrm{CaSiO}_{3}$ melt at $3700{ }^{\circ} \mathrm{C}$ (green dotted line) Fourier transformed over the identical $Q$ range to the experiment with same Lorch modification function. Bottom: Faber-Ziman (Ref. 20) x-ray weighted partial distribution functions for the $\mathrm{CaSiO}_{3}$ melt at $3700{ }^{\circ} \mathrm{C}$ taken directly from the simulation scaled by 0.6 and offset for clarity.

shown in Fig. 2. Previous $\mathrm{x}$-ray measurements on the glass, ${ }^{16,17}$ have established the presence of $\mathrm{SiO}_{4}$ tetrahedra and the $\mathrm{CaO}$ peak at $\sim 2.43 \pm 0.02 \AA$. The structure of the glass at a composition close to $\mathrm{CaSiO}_{3}$ (with the addition of $3 \% \mathrm{Al}_{2} \mathrm{O}_{3}$ ) has been studied in exceptional detail using a double difference neutron isotopic substitution method. ${ }^{4}$ Gaskell et al. ${ }^{4}$ provided evidence that the distribution of calcium atoms was not random in the glass but exhibited a well-defined Ca-Ca peak at $3.8 \AA$ A bearing many characteristics of the crystalline form. The structures of crystalline pyroxenes such as wollastonite can be considered as a close packed oxygen sublattice with $\mathrm{Ca}$ occupying octahedral interstices in alternate layers in a fcc model. This structure has distinguishable domains comprising of sheets of edge shared $\mathrm{CaO}_{6}$ octahedra which are parallel and interleaved with linked $\mathrm{SiO}_{4}$ tetrahedra. Although the recent model by Mead and Mountjoy ${ }^{17}$ and our MD simulations show that the $\mathrm{Ca}$ polymerization in the glass need not be quasicrystalline in nature and branched winding chains of $\mathrm{CaO}$ polyhedra are also consistent with the diffraction data.

\section{MOLECULAR-DYNAMICS SIMULATIONS}

The contributions of the six partial pair distribution functions to the measured $\mathrm{CaSiO}_{3}$ x-ray functions were established using classical MD simulations using potentials based on the point charge Born model. ${ }^{21}$ Partial covalency of the $\mathrm{Si}-\mathrm{O}$ bonds was modeled through the partial charge on the cations and anions. The short range interaction consists of an exponential repulsion term and a dispersion attraction term, in the form of Buckingham

$$
V_{\imath j}(r)=A_{\iota j} \exp \left(-r_{i j} / \rho_{i j}\right)-C_{i j} / r_{i j}^{6},
$$

where $r_{i j}$ is the distance between ion $i$ and $j . A, \rho$, and $C$ are parameters. The potential parameters were obtained by com- 
TABLE I. Atomic charges and Buckingham potential parameters.

\begin{tabular}{lccc}
\hline \hline & $\begin{array}{c}A \\
\text { Pairs }\end{array}$ & $\begin{array}{c}\rho \\
(\mathrm{eV})\end{array}$ & $\begin{array}{c}C \\
(\mathrm{eV} \mathrm{\AA}\end{array}$ \\
\hline $\mathrm{Si}^{2.4}-\mathrm{O}^{-1.2}$ & 13702.905 & 0.193817 & 54.681 \\
$\mathrm{O}^{-1.2}-\mathrm{O}^{-1.2}$ & 2029.2204 & 0.343645 & 192.58 \\
$\mathrm{Ca}^{1.2}-\mathrm{O}^{-1.2}$ & 7747.1834 & 0.252623 & 93.109 \\
\hline \hline
\end{tabular}

bining $a b$ initio calculations with the empirical fitting of related mineral structure and physical property data. This method has been successfully applied to a number of alkali silicate, rare-earth aluminate, and silicate simulations. ${ }^{19,22-27}$ The partial atomic charges and Buckingham potential parameters are shown in Table I.

At short interatomic distances, the Buckingham potential is known to have an unphysical deep potential well that can lead to instabilities of the simulation system. This is caused by the over-riding of the power term to the exponential term at short distances. This was corrected by splicing a power term while maintaining the potential, force, and derivative of force to be continuous at the splicing point. ${ }^{22,26}$ The splicing point was chosen to be where the third derivative of the potential energy was zero. This modification corrects the unphysical potential well at short distance but leaves the original potential unchanged at longer distances. The correction is critical in the simulation of the molten state since the high kinetic energy of ions in the melt can overcome the barrier and reach the deep potential well.

The MD simulations were performed using the constant mass ( $\mathrm{N}$, the number of atoms), pressure $\mathrm{P}$ and temperature $\mathrm{T}$ (NPT) ensemble with $1 \mathrm{fs}$ time steps for 80000 steps. Each cubic simulation cell contained 2500 atoms and with the initial simulation cell size determined based on the experimental density of the glass. At each of the eight simulation temperatures, using the constant mass, volume $\mathrm{V}$ and total energy (NVE) ensemble run of 80000 steps followed the NPT simulations. Structure analyses were obtained by averaging 400 structure snapshots of the final 20000 steps under NVE. For our potential the melting temperature of the model system was $4000 \mathrm{~K}$ so structure factors were extracted at $T_{\mathrm{m}}$, $0.875 T_{\mathrm{m}}, 0.75 T_{\mathrm{m}}$, and $0.625 T_{\mathrm{m}}$ to provide the same effective temperature range as the supercooled liquid experimental data. The simulations confirm that the $2.34 \AA$ peak in the $\mathrm{x}$-ray pattern arises mainly from the $\mathrm{Ca}-\mathrm{O}$ peak and shows a high- $r$ contribution from the O-O correlations. Whereas the main contributions to the $3.50 \AA$ peak comes mainly from the $\mathrm{Ca}-\mathrm{Si}$ and $\mathrm{Ca}-\mathrm{Ca}$ partial pair-distribution functions.

\section{DISCUSSION}

\section{A. High-temperature liquid state}

The concept of diffraction isosbestic points was introduced by Johnson, ${ }^{28}$ to explain the linear dependence of the differential distribution function with concentration in Serich $\mathrm{Si}_{\mathrm{x}} \mathrm{Se}_{(1-\mathrm{x})}$ glasses. Isosbestic points have traditionally been associated with identifying a two-state liquid system

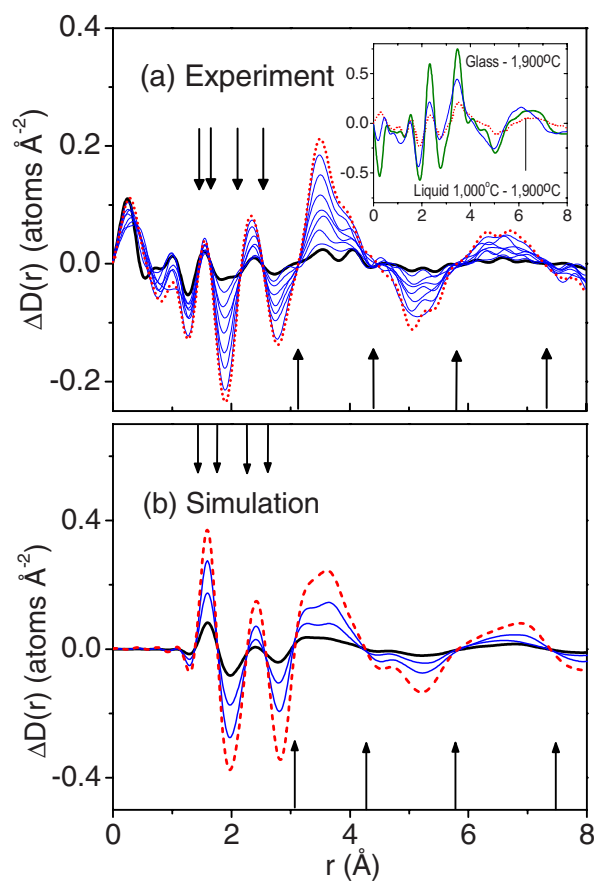

FIG. 3. (Color online) Top: differences between the x-ray experimental liquid $D(r)$ 's held at constant temperatures, for $1033{ }^{\circ} \mathrm{C}$ minus $1900{ }^{\circ} \mathrm{C}$ (red dashed line), and $1800{ }^{\circ} \mathrm{C}$ minus $1900{ }^{\circ} \mathrm{C}$ (thick black solid line). The thin blue lines in between these extremes represent the $D(r)$ differences in $\sim 100{ }^{\circ} \mathrm{C}$ increments [see Fig. 1(a)]. The diffraction isosbestic points are marked with arrows (see text). Inset: shows the $\Delta D(r)$ 's for the liquid at $1033{ }^{\circ} \mathrm{C}$ minus $1900{ }^{\circ} \mathrm{C}$ (dashed red line), $800{ }^{\circ} \mathrm{C}$ minus $1900{ }^{\circ} \mathrm{C}$ (thin blue solid line), and the room-temperature glass minus $1900{ }^{\circ} \mathrm{C}$ (thick green solid line). Bottom: differences between the model liquid $D(r)$ 's taken from the MD simulations for 0.5 minus $T_{\mathrm{m}}$ (red dashed line), $0.625 T_{\mathrm{m}}$ minus $T_{\mathrm{m}}$, and $0.75 T_{\mathrm{m}}$ minus $T_{\mathrm{m}}$ (thin blue lines) $0.875 T_{\mathrm{m}}$ minus $T_{\mathrm{m}}$ (thick solid black line).

using optical spectroscopy. Diffraction isosbestic points are common points in the diffraction pattern and Tse et al. ${ }^{29}$ have extended Johnson's ${ }^{28}$ ideas to argue that a system exhibiting these points as a function of temperature can be interpreted in terms of a two-state model. ${ }^{29}$ We note that since all of our data were Fourier transformed at the identical value of $Q$ $=19 \AA^{-1}$ with a $\operatorname{Lorch}^{30}$ modification function this does not enter into the analysis. Furthermore, although diffraction isosbestic points for $T>1000{ }^{\circ} \mathrm{C}$ are observed directly in the differential distribution functions, $D(r)$, shown in Fig. 2, they become much more apparent in the difference function $\Delta D(r)=D_{\mathrm{T}}(r)-D_{0}(r)$ shown in Fig. 3, where the highest temperature $D_{0}(r)$ at $1900{ }^{\circ} \mathrm{C}$ has been subtracted. Diffraction isosbestic points are observed for liquid $\mathrm{CaSiO}_{3}$ ( $T$ $\left.>1000{ }^{\circ} \mathrm{C}\right)$ at $1.48,1.63,2.15,2.52,3.13,4.47,5.80$, and $7.28 \AA$ A. For calcium silicate liquids we argue that Johnson's clustered unit model ${ }^{28}$ applies in this temperature regime in the form of aggregation of Ca-rich atom regions (by analogy with the compositional dependence in Ref. 28). Based on the peaks in the liquid diffraction data for $\mathrm{CaSiO}_{3}$ this implies the existence of two distinct domains on a length scale of 2-18 ̊⿱: one domain comprising of edge shared $\mathrm{CaO}_{6}$ octahedra and the other a mixture of $\mathrm{CaO}$ polyhedra and $\mathrm{SiO}_{4}$ 


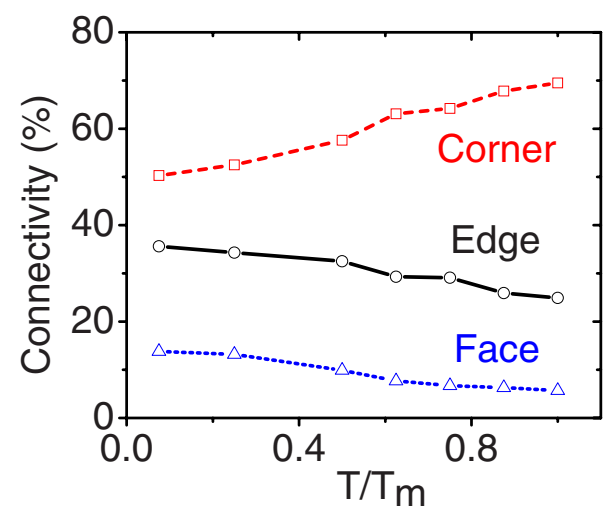

FIG. 4. (Color online) Changes in Ca-polyhedral connectivity of the liquid/glass $\mathrm{MD}$ model of $\mathrm{CaSiO}_{3}$ as a function of temperature.

tetrahedra, these domains are presumably corner shared by oxygen atoms at the interface. For temperatures $T_{\mathrm{g}}<T\left({ }^{\circ} \mathrm{C}\right)$ $<1000$ the diffraction isosbestic points are no longer observed, consistent with the transition from normal to complex behavior around $T=1.2 T_{\mathrm{g}}{ }^{1,2}$ The onset of solid glass like behavior is associated with rapid peak growth associated with $\mathrm{Ca}$ polymerization with decreasing temperature. It is important to note that the existence of isosbestic points do not definitively prove the system is two phase; the sharpening of intramolecular correlations in a molecular liquid upon cooling for example, could also produce the same effect due to narrowing zero point motions. However, strong arguments for the heterogeneous clustered unit model can be made due to the observation of the increased number of edge shared $\mathrm{CaO}$ octahedra upon cooling liquid $\mathrm{CaSiO}_{3}$ and because of the supporting evidence from MD simulations (see Fig. 3).

The MD model shows that at the melting temperature a few threefold and fivefold coordinated Si exist but $98.5 \%$ of these polyhedra remain corner shared and only $1.5 \%$ are edge shared. By $0.75 T_{\mathrm{m}}$ all the $\mathrm{SiO}_{4}$ units are corner shared and in the room-temperature glass all $\mathrm{Si}$ ions are tetrahedrally coordinated by oxygen. The MD model shows that the number of edge and face shared $\mathrm{Ca}$ polyhedra increase steadily with decreasing temperature indicating Ca polymerization occurs upon supercooling the melt, see Fig. 4.

\section{B. Below $1.2 T_{\mathrm{g}}$}

The main local structural changes that occur upon cooling the melt are at 2.34 and $3.50 \AA$ in the x-ray differential distribution function (Fig. 3) and we interpret the growth of these peaks upon cooling as the increase in size of edgeshared octahedral clusters or chains. Integrating these two peaks in the $\Delta D(r)$ functions between associated isosbestic points we can obtain an estimate of the rate of growth of the $\mathrm{CaO}_{6}$ and $\mathrm{Ca}-\mathrm{Ca}$ edge-sharing octahedral units in the melt as a function of temperature, see Fig. 4. The same integration limits were also used below $1000{ }^{\circ} \mathrm{C}$ for consistency although the isosbestic points are no longer present introducing a small error. Given that, for both peaks the integrals can be fitted using a linear term at high temperatures and a nonlinear term at low temperatures. This analysis implies that there is a linear growth of chains and/or (possibly) sheets of

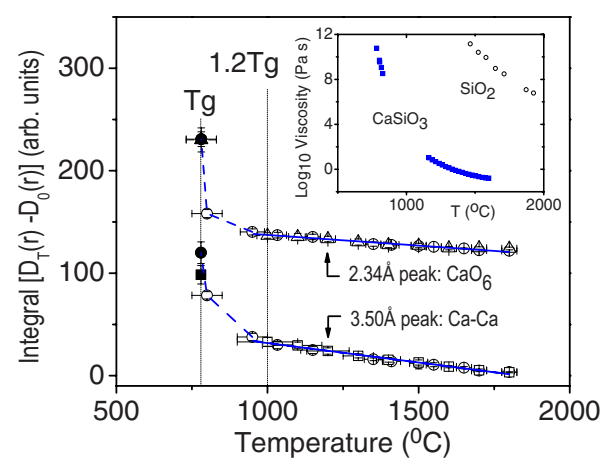

FIG. 5. (Color online) The integrals of the differences between the static state differential distribution functions, $\Delta D(r)$ measurements (see text), shown in Fig. 3 (top) for liquid $\mathrm{CaSiO}_{3}$. The integrals were taken over the region between the isosbestic points for the 2.34 and $3.50 \AA$ peaks in $\Delta D(r)$. The symbols represent different experimental runs. Both curves are fitted with a linear (blue dashed) line which has been extended to lower temperatures by a dashed blue line (see Fig. 6 for more extensive measurements in this region). The glass measured at $25^{\circ} \mathrm{C}$ was taken as a representative liquid structure at $T_{g}=781{ }^{\circ} \mathrm{C}$ for $\mathrm{CaSiO}_{3}$ (Ref. 31) (solid symbols). The $1.2 T_{g}$ line is calculated in Kelvin but shown in degrees Celsius. Inset: viscosity-temperature relation of liquid $\mathrm{CaSiO}_{3}$ (blue squares) (Refs. 10 and 11) and $\mathrm{SiO}_{2}$ (circles) (Ref. 32).

$\mathrm{CaO}_{6}$ in the liquid between 1900 and $1000{ }^{\circ} \mathrm{C}$ but the aggregation increases more rapidly as the glass transition temperature is approached and is concomitant with a steep rise in viscosity (Fig. 5). Similar curves were obtained for the data for all the glass forming compositions studied but only linear behavior is observed for the high $\mathrm{CaO}$ content samples which could not be vitrified.

\section{Fast-cooling experiments}

In order to probe the low-temperature region a separate set of experiments were performed using the area detector. The $\mathrm{CaSiO}_{3}$ melts were quenched by switching the laser off and sequential measurements were taken every $200 \mathrm{~ms}$. The results shown in Fig. 6 around $1000{ }^{\circ} \mathrm{C}$ are therefore intriguing since they suggest the small deviation in structure between the isothermal and fast quenched $\mathrm{CaSiO}_{3}$ samples which begins at $\sim 1100{ }^{\circ} \mathrm{C}$ is real.

The fast cooling measurements also show a distinct plateau as $T_{\mathrm{g}}$ is approached that is absent in the slow-cooling measurements. This difference is attributed to the higher fictive temperature expected in the quenched sample compared to one that is vitrified by slow cooling, i.e., different thermal histories. The differences observed in the present work also provide a possible explanation of the differences in structure measured in prior work by Taniguchi et al. ${ }^{16}$ and Mead and Moutnjoy. ${ }^{17}$ Further fast-cooling structural studies are planned on other systems to see if these features are ubiquitous in quenched glass-forming liquid oxides.

\section{SUMMARY}

Static and fast cooling high-energy synchrotron x-ray structure factor measurements have been performed on aero- 


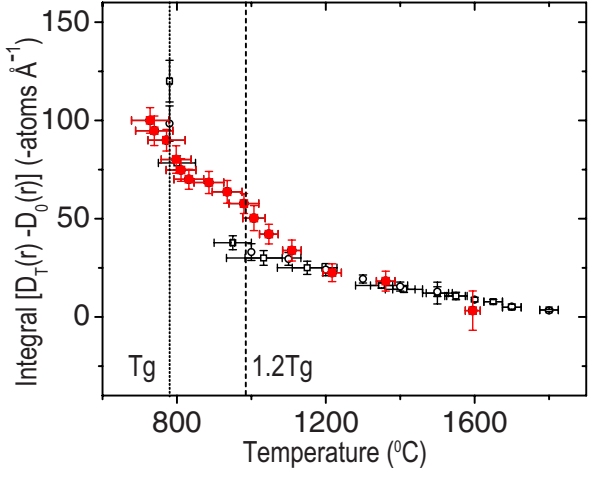

FIG. 6. (Color online) The integrals of the differences between the differential distribution functions, $\Delta D(r)$, for liquid $\mathrm{CaSiO}_{3}$. The integrals were taken over the region between the isosbestic points for the $3.50 \AA \mathrm{Ca}-\mathrm{Ca}$ peak in $\Delta D(r)$. The fast cooling measurements (solid red squares) are compared to isothermal measurements (open symbols from Fig. 5). The dotted line represents $T_{g}$ (Ref. 31) and the dashed line $1.2 T_{g}\left(992{ }^{\circ} \mathrm{C}\right)$. The $1.2 T_{g}$ line is calculated in Kelvin but shown in degrees Celsius for consistency.

dynamically levitated $\mathrm{CaSiO}_{3}$ droplets. Upon cooling the high-temperature liquid, diffraction isosbestic points are observed in the pair-distribution function. By comparison with molecular-dynamics simulations and glass structural data it is argued that the experimental results are consistent with the existence of a heterogeneous liquid state. The primary structural changes upon cooling being associated with the polymerization of edge-shared $\mathrm{Ca}$ octahedra, which is linear in the high-temperature melt but exhibits rapid growth below $1.2 T_{\mathrm{g}}$. The heterogeneous liquid interpretation is supported by the MD simulations which show the $\mathrm{CaSiO}_{3}$ glass has $10 \%$ more edge-shared polyhedra and $20 \%$ fewer corner

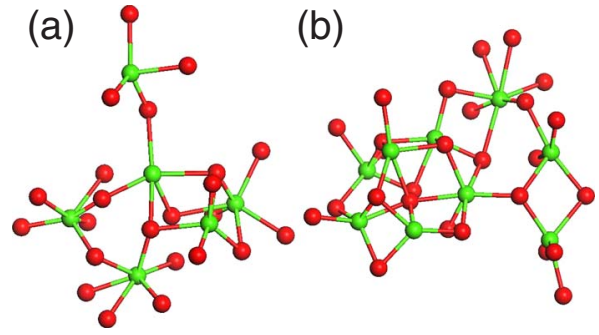

FIG. 7. (Color online) Molecular-dynamics snapshots of the polymerization of $\mathrm{CaO}$ polyhedra from the predominantly corner shared $\mathrm{CaSiO}_{3}$ equilibrium melt (a) to more edge-shared $\mathrm{CaO}_{6}$ units in the glass (b) obtained from MD simulations. The $\mathrm{Ca}$ ions are shown in green (light) and $\mathrm{O}$ in red (dark).

shared polyhedra than the liquid model, as illustrated in Fig. 7. In the future, it is anticipated that the ability to probe metastable structures of fragile melts near $T_{\mathrm{g}}$ using a levitator ${ }^{33}$ together with high-flux x-ray diffraction with fast, high efficiency two-dimensional detectors and rapid data acquisition, will help clarify the relationship between dynamic and structural heterogeneity in supercooled liquids. ${ }^{3}$

\section{ACKNOWLEDGMENTS}

We appreciate the efforts of $\mathrm{W}$. Woerner in performing the probe analyses, and for access to infrastructure provided by H. Nekvasil and D. H. Lindsley at Stony Brook used for preparing the precursor materials for this study. This work was supported by the U.S. DOE, at Argonne National Laboratory under Contract No. DE-AC02-06CH11357, by NSF under Grant No. DMR-0800415, and NSF under Grants No. DMR-0907593 and No. DE-SC0002510. J.B.P. is grateful to the UK Fulbright Program for support during a sabbatical stay at Edinburgh University.
*Corresponding author

${ }^{1}$ M. Goldstein, J. Chem. Phys. 51, 3728 (1969).

${ }^{2}$ M. D. Ediger, Annu. Rev. Phys. Chem. 51, 99 (2000).

${ }^{3}$ H. Sillescu, J. Non-Cryst. Solids 243, 81 (1999).

${ }^{4}$ P. H. Gaskell, M. C. Eckersley, A. C. Barnes, and P. Chieux, Nature (London) 350, 675 (1991).

${ }^{5}$ G. N. Greaves, A. Fontaine, P. Lagarde, D. Raoux, and S. J. Gurman, Nature (London) 293, 611 (1981).

${ }^{6}$ S. Soyer Uzun, S. Sen, C. J. Benmore, and B. G. Aitken, J. Phys.: Condens. Matter 20, 335105 (2008).

${ }^{7}$ T. Fujita, K. Konno, W. Zhang, V. Kumar, M. Matsuura, A. Inoue, T. Sakurai, and M. W. Chen, Phys. Rev. Lett. 103, 075502 (2009).

${ }^{8}$ B. O. Mysen, D. Virgo, and F. A. Seifert, Rev. Geophys. 20(3), 353 (1982).

${ }^{9}$ Q. Mei, C. J. Benmore, and J. K. R. Weber, Phys. Rev. Lett. 98, 057802 (2007).

${ }^{10}$ J. O. Bockris and D. C. Lowe, Proc. R. Soc. London, Ser. A 226, 423 (1954).

${ }^{11}$ M. Solvang, Y.-Z. Yue, S. L. Jensen, and D. B. Dingwell, J. Non-Cryst. Solids 351, 499 (2005).
${ }^{12}$ Q. Mei, C. J. Benmore, J. K. R. Weber, M. Wilding, J. Kim, and J. Rix, J. Phys.: Condens. Matter 20, 245107 (2008).

${ }^{13}$ A. P. Hammersley, S. O. Svensson, M. Hanfland, A. N. Fitch, and D. Hausermann, High Press. Res. 14, 235 (1996).

${ }^{14}$ X. Y. Qiu, J. W. Thompson, and S. J. L. Billinge, J. Appl. Crystallogr. 37, 678 (2004).

${ }^{15}$ Y. Waseda and J. M. Toguri, Metall. Trans. B 8, 563 (1977).

${ }^{16}$ T. Taniguchi, M. Okuno, and T. Matsumoto, J. Non-Cryst. Solids 211, 56 (1997).

${ }^{17}$ R. N. Mead and G. Mountjoy, J. Phys. Chem. B 110, 14273 (2006).

${ }^{18}$ M. C. Wilding, C. J. Benmore, and J. K. R. Weber, J. Mater. Sci. 43, 4707 (2008).

${ }^{19}$ J. Du, J. Am. Ceram. Soc. 92, 87 (2009).

${ }^{20}$ D. Keen, J. Appl. Crystallogr. 34, 172 (2001).

${ }^{21}$ A. N. Cormack and J. Du, J. Non-Cryst. Solids 293-295, 283 (2001).

${ }^{22}$ J. Du and A. N. Cormack, J. Non-Cryst. Solids 349, 66 (2004).

${ }^{23}$ J. Du and A. N. Cormack, J. Am. Ceram. Soc. 88, 2532 (2005).

${ }^{24}$ J. Du and L. R. Corrales, Phys. Rev. B 72, 092201 (2005).

${ }^{25}$ J. Du and A. N. Cormack, J. Non-Cryst. Solids 351, 2263 
(2005)

${ }^{26}$ J. Du and L. R. Corrales, J. Non-Cryst. Solids 352, 3255 (2006).

${ }^{27}$ J. Du, C. Benmore, L. R. Corrales, R. Hart, and R. Weber, J. Phys.: Condens. Matter 21, 205102 (2009).

${ }^{28}$ R. W. Johnson, J. Non-Cryst. Solids 88, 366 (1986).

${ }^{29}$ J. S. Tse, D. D. Klug, M. Guthrie, C. A. Tulk, C. J. Benmore, and J. Urquidi, Phys. Rev. B 71, 214107 (2005).

${ }^{30}$ E. A. Lorch, J. Phys. C 2, 229 (1969).
${ }^{31}$ M. Micoulaut, M. Malki, P. Simon, and A. Canizares, Philos. Mag. 85, 3357 (2005).

${ }^{32} \mathrm{G}$. Urbain, Y. Bottinga, and P. Richet, Geochim. Cosmochim. Acta 46, 1061 (1982).

${ }^{33}$ L. Hennet,I. Pozdnyakova, A. Bytchkov, D. L. Price, G. N. Greaves, M. Wilding, S. Fearn, C. M. Martin, D. Thiaudière, J.-F. Bérar, N. Boudet, and M.-L. Saboungi, J. Chem. Phys. 126, 074906 (2007). 